Original

\title{
Aproximación a un modelo de costo eficacia de protectores auditivos en el ambiente laboral
}

\section{Approach to a cost-effectiveness model of hearing protectors in the workplace}

\author{
Ivonne Valero-Pacheco', Martha Isabel Riaño-Casallas², Frady Rodríguez-Páez ${ }^{3}$
}

1. Profesora Programa Especialización en Gestión de la Seguridad y Salud en el Trabajo. Universidad Jorge Tadeo Lozano. Bogotá - Colombia.

2. Profesora Programa Especialización en Gestión de la Seguridad y Salud en el Trabajo. Universidad Jorge Tadeo Lozano.Bogotá - Colombia E-mail: marthai.rianoc@utadeo.edu.co.

3. Coordinador Académico Programa Especialización Gerencia y Auditoría de la Calidad en Salud. Universidad Jorge Tadeo Lozano. Bogotá - Colombia E-mail: fredyg.rodriguezp@utadeo.edu.co.

Recibido: 06-12-13

Aceptado: 23-05-14

\section{Correspondencia}

Ivonne Constanza Valero Pacheco

Universidad de Bogotá Jorge Tadeo Lozano

Carrera 4 No. 22-61 Módulo 16 Edificio de Postgrados Oficina 301. Colombia

Correo electrónico: ivonne.valero@utadeo.edu.co

Teléfono: (571) 2427030 Extensión 3684

Resumen

Introducción: La escasa información disponible en relación con modelos de evaluación de costo efectividad de la protección auditiva, para la prevención de la hipoacusia neurosensorial inducida por el ruido laboral en Colombia, así como la confiabilidad de la información del nivel de atenuación de ruido que es suministrada por los fabricantes de elementos de protección auditiva, justificaron el desarrollo de esta investigación.

Objetivos: Proponer un modelo de evaluación de costo eficacia de protectores auditivos ofertados en el mercado colombiano, frente a las necesidades de atenuación contra el ruido establecidas por la legislación colombiana.

Materiales y Métodos: Se elaboró un modelo de costo eficacia de protectores auditivos en Colombia. Para ello se seleccionó del total de la oferta existente en el mercado colombiano un protector tipo copa y otro tipo inserción, a los cuales se les aplicó la metodología ANSI S3.19 para determinar el Nivel de Reducción del Ruido (NRR). En la prueba participaron 21 sujetos. En el modelo se consideraron variables como el costo equivalente anual, el nivel de atenuación real y la relación costo eficacia.

Resultados: El nivel de atenuación del ruido suministrado en las fichas técnicas del fabricante difiere del encontrado en la prueba realizada para cada uno de los protectores, en el caso de los de tipo copa su eficacia es del 37\% menos de lo esperado y para los de inserción del 59\%. El cálculo de la Razón de Costo Efectividad (RCE) para los protectores tipo copa muestra un valor de $\$ 90.055$ por cada unidad de eficacia, mientras que la RCE de los protectores de inserción es de $\$ 465.034$.

Discusión/Conclusiones: Aunque las diferencias en el nivel de eficacia observado pueden estar afectadas por factores humanos, la metodología de la prueba y las características propias del protector auditivo, estos hallazgos son consistentes con la recomendación de National Institute for Occupational Safety and Health (NIOSH) sobre la reducción del NRR. 
Es importante considerar la aplicación de modelos de costo eficacia para la selección y adquisición de elementos de protección personal en las empresas, lo que lleva incorporar una política de protección al trabajador coherente con los recursos disponibles en la empresa para tal fin.

Med Segur Trab (Internet) 2014; 60 (235) 313-321

Palabras clave: Tapones de oído, dispositivos de protección de la audición, ruido, evaluación de costoefectividad, análisis costo-beneficio.

Abstract:

Introduction: In Colombia the poor information available of cost effectiveness assessment models of hearing protection to prevent work noise-induced sensorineural hearing loss, as well as the reliability of information on the level of Noise Reduction Rating (NRR) that is provided by the manufacturers of hearing protectors is the justification for development of this research.

Objectives: The aim of this study is proposed a model for evaluating cost-effectiveness of hearing protectors in the Colombian market according to needs against noise attenuation provided by Colombian laws.

Materials and Methods: In the study was designed a model of cost effectiveness of hearing protectors in Colombia. For this purpose would selected one earmuffs and one earplugs offered in the Colombian market. The methodology applied was ANSI S3.19 to determine the NRR. The trial involved 21 subjects. The model considered variables as the annual equivalent cost, the real attenuation level and the Cost Effectiveness ratio.

Results: Noise Reduction Rating provided by the manufacturers technical specifications differ from the results found in the trial. In earmuffs was 37\% less than expected and earplugs was 59\%. The CER for earmuffs was COP $\$ 90,055$ for one unit of effectiveness, while CER earplugs was COP $\$ 465,034$.

Discussion/Conclusions: Differences in the level of efficacy observed may be affected by human factors, the trial methodology and the characteristics of the hearing protector; however these findings are consistent with the NIOSH recommendation on reducing NRR.

It is important to consider application of cost-effectiveness models for the selection and buy personal protective equipment in enterprises, leading to incorporate a worker protection policy according to the resources available in the company.

Med Segur Trab (Internet) 2014; 60 (235) 313-321

Key words: ear protective devices, noise, cost-effectiveness evaluation, cost-benefit analysis. 


\section{INTRODUCCIÓN}

Las organizaciones desde su origen están en la búsqueda de crecer, mantenerse en el mercado y generar utilidades, sin embargo, y acorde con la evolución económica y social se han desarrollado normas y políticas que en el contexto internacional se enfocan a la protección de los trabajadores con fines orientados a mejorar sus condiciones laborales y de salud. Es así, como la Organización Internacional del Trabajo (OIT) ha promovido la política de trabajo decente, que incluye recomendaciones frente a la prevención de la enfermedad laboral y el accidente de trabajo, bajo el cual la OIT ha diseñado el modelo de sistema de gestión de seguridad y salud en el trabajo. Uno de los principales propósitos de este sistema es "proteger a los trabajadores contra los peligros y eliminar las lesiones, enfermedades, dolencias, incidentes y muertes relacionadas con el trabajo" (1). Esta premisa que aplica al orden nacional se adapta a las organizaciones de acuerdo con sus necesidades.

En la medida que las organizaciones buscan su rentabilidad uno de los factores que afecta negativamente a la misma son los costos asociados al no control del riesgo laboral que conlleva a la aparición de lesiones relacionadas con el trabajo. Como parte de la gestión de estos riesgos, la selección, compra y suministro de los elementos de protección individual o personal es uno de los tantos mecanismos de control frecuentemente utilizados. No obstante, la decisión de la selección y compra de tales elementos depende de aspectos tanto técnicos como económicos. En este sentido, las evaluaciones económicas son una herramienta que permite una mejor toma de decisiones basada en la medición de diferentes alternativas en cuanto a la efectividad y los costos (2). Para el caso de la priorización de los elementos de protección personal, las evaluaciones de costo efectividad son una alternativa que evita las discusiones éticas asociadas a la monetización de los resultados en salud (3).

La hipoacusia neurosensorial inducida por ruido, entendida ésta como el resultado de la exposición prolongada a ruido en el lugar de trabajo (7), es una de las principales enfermedades laborales a nivel mundial, y para el caso de Colombia representa el cuarto de los diagnósticos calificados de origen laboral (8). Para la prevención de esta enfermedad los métodos habituales de control de atenuación del ruido se dan en la fuente, el medio y en la persona; para este último caso, el uso de tapones de oído tanto de copa como de inserción son los mecanismos de control que cumplen con tal finalidad.

Teniendo en cuenta lo anterior, y ante la poca evidencia frente a estudios de la costo eficacia de este tipo de elementos, la investigación desarrollada plantea como finalidad proponer un modelo de evaluación de la costo efectividad de protectores auditivos ofertados en el mercado colombiano frente a la necesidades de atenuación contra el ruido establecidas por la legislación colombiana.

\section{MODELO DE COSTO EFECTIVIDAD EN SALUD LABORAL}

Las empresas en la búsqueda de tomar las mejores decisiones para el bienestar de los trabajadores en el marco de las restricciones presupuestales, deben evaluar tanto los beneficios como los costos de diferentes alternativas para el control del ruido ocupacional en la persona. Dentro de la oferta de protectores auditivos en Colombia, se identifican múltiples marcas y referencias que presentan en sus fichas técnicas diferentes niveles de atenuación del ruido (efectividad) y a su vez, tienen precios disímiles. Para poder realizar una comparación de la efectividad y los costos entre los diferentes tipos de protectores, se utilizan los análisis de costo efectividad como una de las herramientas que contribuye a fundamentar la toma de decisiones en razón a que existe un resultado de interés común, que es la atenuación del ruido como mecanismo de la prevención de la enfermedad laboral. 
Si bien es cierto, que la lógica en la toma de decisiones de los empleadores es más afín con los análisis de costo beneficio, en materia de salud, y particularmente de la salud de los trabajadores, el empleador más allá de su perspectiva financiera tiene el deber de proteger a sus trabajadores frente a cualquier contingencia derivada del trabajo por cuanto se constituye como un principio fundamental garantizar las condiciones dignas y justas de trabajo (4) (5). Por lo anterior, para este estudio, se utiliza el modelo de Costo Efectividad (CE), el cual permite una discriminación cuantitativa de las variables de costos y efectos de una intervención, al compararlos con los costos y efectos de otra intervención para cualquier problema o problemas de salud cuyos resultados se expresen en las mismas unidades (2) (6).

La aplicación del modelo de CE al caso de estudio relacionado con la atenuación de ruido laboral como uno de los mecanismos de prevención de la hipoacusia neurosensorial inducida por ruido, tiene en cuenta como unidad de medida de la efectividad el Noise Reduction Rating o Nivel de Reducción del Ruido (NRR), y para la medición de los costos, el precio de los protectores auditivos en el mercado colombiano.

\section{EFECTIVIDAD DE LA PROTECCIÓN AUDITIVA}

El ruido es un sonido no deseado que tiene como características la intensidad (volumen medido en decibeles), el tono (frecuencia) y el timbre (fuente emisora), y de acuerdo con su distribución temporal se puede clasificar en continuo estable, continuo fluctuante, intermitente y de impacto o impulso (9). El ruido puede ser peligroso para la salud debido tanto a la duración de la exposición como por sus niveles (10), los cuales en el ambiente laboral tienen como fuente emisora máquinas, equipos, falta de aislamientos acústicos, falta de sistemas de amortiguación, entre otros (9).

En relación con los métodos de control del ruido ocupacional en la persona, se incluyen aquellos que taponan el canal auditivo y los que cubren la totalidad del pabellón auditivo, denominados respectivamente protectores auditivos de inserción y de copa o tipo orejeras. De cada uno de estos tipos de protectores existen en el mercado múltiples referencias de acuerdo con el material, diseño y nivel de atenuación, entre otros aspectos.

La atenuación del ruido se define como la diferencia en decibeles entre el umbral de audición con y sin protector auditivo ante un estímulo dado al sujeto a prueba (11). Para efectos de la medición de los niveles de atenuación de ruido existen diversos métodos propuestos por entidades como ISO, ANSI, y para el caso de Colombia Icontec, los cuales son adoptados de manera autónoma por los fabricantes de elementos de protección auditiva.

\section{MATERIAL Y MÉTODOS}

Para el desarrollo de la investigación se partió del conocimiento de los protectores auditivos ofertados en Colombia a través de la realización de un inventario de tales elementos. En este se incluyeron nivel de atenuación, vida útil, características técnicas y precio. Del inventario se tomó como criterio para la selección la referencia con mayor de nivel de atenuación reportado en la ficha técnica, tanto para el conjunto de protectores de copa como para los de inserción.

Por otra parte, se analizaron las normas técnicas de referencia tales como ISO 4859 , NTC 5629. ANSI S3.19, NTC 2272. Para efectos de la aplicación del método, se escogió la Norma ANSI S3.19 puesto que es la que está referida en la mayoría de las fichas técnicas de los protectores revisados.

De acuerdo con el método, participaron 24 voluntarios, conformada por hombres y mujeres, entre los 20 a 40 años, laboralmente activa, de los cuales 21 cumplieron con un diagnóstico auditivo normal (audición bilateral normal) y continuaron con la prueba. La 
totalidad de los participantes fue informada sobre la finalidad del estudio y los posibles riesgos derivados de ella, y manifestaron por escrito el consentimiento para su participación. Se realizó audiometría tonal aérea en cabina sonoamortiguada, con audiómetro previamente calibrado, para determinar el umbral mínimo de audición de cada individuo en las frecuencias de 250, 500, 1000, 2000, 3000, 4000 y $6000 \mathrm{~Hz}$.

Para cada Banda de Octava y para cada individuo, se determinó la atenuación como la diferencia entre el umbral de audición con y sin protector auditivo. Posteriormente, se halló la media y la desviación típica de las atenuaciones obtenidas en los individuos para cada banda de octava, para determinar el valor medio menos la desviación típica, ambos valores obtenidos en la prueba, y para obtener los resultados finales del NRR se aplica la metodología según la norma OSHA-29CFR - 1910.95 App: Método para estimar la atenuación del protector auditivo (9). variables:

Para el desarrollo del modelo de costo efectividad se consideraron las siguientes

Costo: Precio unitario en punto de distribución comercial sin descuentos por volumen. En pesos colombianos (COP) al momento de la realización del estudio.

Vida Útil: Duración estimada en días que puede tener el protector auditivo cumpliendo correctamente con su función según el reporte de las fichas técnicas, en caso de encontrarse este dato se supone que los protectores de inserción desechables tienen vida útil máxima de un día, mientras que los protectores de copa como mínimo duran un año (12).

- Cantidad anual: Cantidad de protectores auditivos que se deben adquirir en un año, cociente entre vida útil y 292 días laborales calculados teniendo en cuenta la jornada laboral ordinaria según la normatividad laboral colombiana.

- Costo equivalente anual: Costo unitario por vida útil expresada en años. Es decir, costo de los protectores auditivos utilizados durante un año. En pesos colombianos (COP) al momento de la realización del estudio.

- Atenuación: Corresponde al Nivel de reducción de ruido (NRR) que aparece en la ficha técnica del protector auditivo.

- Atenuación real: El resultado de la prueba aplicada a las referencias seleccionadas en este estudio.

- Eficacia: Valor máximo de atenuación posible para protectores auditivos resultado de la prueba bajo condiciones controladas.

- RCE: Razón de Costo Eficacia, informa del costo por unidad de eficacia. En pesos colombianos (COP) al momento de la realización del estudio.

\section{RESULTADOS}

Partiendo de la oferta de protectores auditivos en el mercado colombiano se encontraron 6 marcas de dispositivos tipo inserción y 8 de tipo copa, cada una con un número variable de referencias. En la Tabla 1 se observa el listado de marcas y referencias con el rango de NRR para cada una. Para efectos de confidencialidad de la información se le asignó aleatoriamente una letra a cada marca. 
Tabla I. Inventario de protectores auditivos

\begin{tabular}{cccc}
\hline Tipo de protector & Marcas & $\begin{array}{c}\text { Número de } \\
\text { referencias } \\
\text { por marca }\end{array}$ & Rango de NRR* por referencias \\
\hline A & 4 & $21 \mathrm{~dB}-33 \mathrm{~dB}$ \\
Inserción & B & 9 & $25 \mathrm{~dB}-32 \mathrm{~dB}$ \\
& C & 1 & $26 \mathrm{~dB}$ \\
& D & 9 & $28 \mathrm{~dB}-36 \mathrm{~dB}$ \\
E & 8 & $26 \mathrm{~dB}-33 \mathrm{~dB}$ \\
& F & 3 & $25 \mathrm{~dB}-$ otros no brindan ficha técnica \\
\hline & A & 7 & $20 \mathrm{~dB}-34 \mathrm{~dB}$ \\
B & 2 & $21 \mathrm{~dB}-27 \mathrm{~dB}$ \\
Copa & 8 & 7 & $26 \mathrm{~dB}-34 \mathrm{~dB}$ \\
& D & 3 & $24 \mathrm{~dB}-35 \mathrm{~dB}-26 \mathrm{~dB}$ \\
& E & 8 & $23 \mathrm{~dB}-30 \mathrm{~dB}$ \\
& F & 1 & $26 \mathrm{~dB}$ \\
& G & 3 & $23 \mathrm{~dB}-28 \mathrm{~dB}$ \\
\hline
\end{tabular}

*NRR: Noise Reduction Rating

A partir de los datos de NRR especificados en la ficha técnica de marcas y referencias, y como se indicó en la metodología se seleccionó un protector evaluar de tipo copa y uno tipo inserción, cuyas características de atenuación se presentan en las tablas 2 y 3 :

Tabla II. Datos suministrados por el fabricante del protector auditivo tipo copa seleccionado

\begin{tabular}{lcccccccc}
\hline Frecuencias de octava en HZ & 250 & 500 & 1000 & 2000 & 3000 & 4000 & 6000 & NRR \\
\cline { 1 - 7 } Protector Atenuación en dB & 26.0 & 36.6 & 40.6 & 38.0 & Sin dato & 42.7 & 41.7 & \\
\cline { 1 - 7 } Desviación Estándar SD & 2.3 & 2.3 & 2.4 & 2.5 & Sin dato & 1.8 & 2.1 & \\
\hline
\end{tabular}

Tabla III. Datos suministrados por el fabricante del protector auditivo tipo inserción seleccionado

\begin{tabular}{lcccccccc}
\hline Frecuencias de octava en HZ & 250 & 500 & 1000 & 2000 & 3000 & 4000 & 6000 & NRR \\
\hline Protector Inserción & 40.9 & 44.8 & 43.8 & 36.3 & Sin dato & 42.6 & Sin dato & \\
\cline { 1 - 6 } Desviación Estándar SD & 5.0 & 3.3 & 3.6 & 4.9 & Sin dato & 3.1 & Sin dato & \\
\hline
\end{tabular}

A los protectores auditivos seleccionados se les realizó la evaluación indicada en la metodología y se obtuvieron los siguientes resultados:

Tabla IV. Cálculos de atenuación con octavas del protector auditivo tipo copa

\begin{tabular}{lrrrrrrrr}
\hline Frecuencias de octava en HZ & 250 & 500 & 1000 & 2000 & 3000 & 4000 & 6000 & NRR \\
\hline Protector COPA & 22.38 & 34.76 & 40.00 & 32.86 & 36.19 & 36.43 & 35.48 & \\
\cline { 1 - 7 } Desviación Estándar SD & 9.83 & 10.06 & 5.92 & 6.81 & 6.10 & 3.92 & 8.20 & \\
\hline
\end{tabular}


Tabla V. Cálculos de atenuación con octavas del protector auditivo tipo inserción

\begin{tabular}{lcccccccc}
\hline Frecuencias de octava en HZ & 250 & 500 & 1000 & 2000 & 3000 & 4000 & 6000 & NRR \\
\cline { 1 - 7 } Protector Inserción & 17.38 & 20.24 & 20.95 & 30.29 & 39.29 & 40.48 & 43.81 & \\
\cline { 1 - 8 } Desviación Estándar SD & 10.44 & 8.87 & 8.00 & 8.35 & 8.41 & 10.83 & 9.61 & \\
\hline
\end{tabular}

El cálculo de la RCE se presenta en la tabla 6, en donde se observa que el costo unitario de los protectores auditivos tipo copa es alto en relación con los de tipo inserción, el costo equivalente anual es tres veces mayor en los de tipo inserción dada la vida de útil que implica un alto consumo anual de este tipo de elemento. En relación con la eficacia se encontró que el protector tipo copa es 53\% más eficaz que el protector tipo inserción. El cálculo de la RCE para los protectores tipo copa muestra un valor de $\$ 90.055$ por cada unidad de eficacia, mientras que la RCE de los protectores de inserción es de \$465.034.

Tabla VI. Cálculo de la RCE de los protectores auditivos evaluados

\begin{tabular}{lcccccccc}
\hline Tipo de protector & $\begin{array}{c}\text { Costo } \\
\text { Unitario } \\
\text { (COP) }\end{array}$ & $\begin{array}{c}\text { Vida útil } \\
\text { (días) }\end{array}$ & $\begin{array}{c}\text { Cantidad } \\
\text { anual }\end{array}$ & $\begin{array}{c}\text { Costo } \\
\text { equivalente } \\
\text { anual (COP) }\end{array}$ & Atenuación & $\begin{array}{c}\text { Atenuación } \\
\text { real }\end{array}$ & Eficacia & RCE(COP) \\
\hline Tipo Copa & $\$ 57,035$ & 292 & 1 & $\$ 57,035.00$ & 30 & 19 & 0.63 & $\$ 90,055.26$ \\
\hline Tipo Inserción & $\$ 659$ & 1 & 292 & $\$ 192,428.00$ & 29 & 12 & 0.41 & $\$ 465,034.33$ \\
\hline
\end{tabular}

\section{DISCUSIÓN}

El nivel de atenuación del ruido suministrado en las fichas técnicas del fabricante difiere del encontrado en la prueba realizada para cada uno de los protectores, en el caso de los de tipo copa su eficacia es del $37 \%$ menos de lo esperado y para los de inserción del 59\%. Las posibles causas de esta diferencia pueden estar asociadas a factores humanos, a la metodología de la prueba y a las características propias del protector auditivo. En lo que se refiere a las características del protector auditivo (13), la generación de incomodidad está relacionado con la mala elección de éste y la calidad del material.

Respecto a los factores humanos pueden intervenir el nivel de atención del participante y la fatiga en el proceso de las pruebas, como lo menciona la Norma técnica de Prevención (NTP) 284, en donde se confirma que entre otros aspectos la simulación de otras enfermedades y la falta de colaboración pueden generar error en la prueba audiométrica. (14)

Por otra parte, en relación con la metodología de la prueba se tiene la subjetividad de la audiometría, es decir que para ambos factores se pueden ver diferentes respuestas del mismo participante en una misma frecuencia (4) (15).

En cuanto a las características propias del protector auditivo, para el caso de los protectores tipo copa están tanto el tamaño como la forma de la cabeza y el nivel de confort; para los protectores tipo inserción los elementos que pueden influir en la atenuación son su forma, que puede no ser cómoda para el sujeto, la variación en tamaño del canal auditivo externo para cada persona, los materiales de fabricación, los cuales pueden generar alergias o picazón entre otros, y la correcta colocación de estos en el canal auditivo (13). Sin embargo, cabe resaltar que en la prueba la colocación de los protectores auditivos fue hecha por parte de los evaluadores a cada uno de los participantes para asegurar su adecuado uso.

Por otra parte, y en lo concerniente a la aplicación de la metodología para determinar la atenuación del ruido NIOSH recomienda reducir el NRR que proporciona el fabricante en un $25 \%$ para el caso de los protectores tipo copa y en un $70 \%$ para los de inserción (9). Los hallazgos de este estudio son consistentes con esta recomendación. 
Continuando con el objeto del estudio, los resultados para esta investigación frente al modelo de costo-eficacia muestran que el precio unitario del protector tipo inserción es más económico en comparación con los de tipo copa, sin embargo, para la persona encargada de tomar la decisión de compra de los elementos de protección auditiva es importante considerar el costo equivalente anual, sumado a los factores técnicos, que no son objeto de este estudio, pero que deben ser analizados por el área de seguridad y salud en el trabajo.

Lo que demuestra este estudio es la importancia de aplicar modelos de costo eficacia para la selección y adquisición de elementos de protección personal en las empresas, ya que permite analizar variables no solo económicas sino también factores asociados a la eficacia del elemento lo que lleva incorporar una política de protección al trabajador coherente con los recursos disponibles en la empresa para tal fin, buscando la sostenibilidad de la gestión de seguridad y salud en el trabajo en la organización (3).

Los resultados de este estudio evidencian la existencia de una problemática en relación con la efectiva protección auditiva en los trabajadores, asociada tanto a la información pública suministrada por los fabricantes de elementos de protección auditiva, como a los criterios utilizados por los empleadores en la compra de dichos elementos. Ante tal situación, se hace necesario ampliar el alcance del tamaño de la muestra de participantes así como el incluir un número mayor de marcas y referencias de cada tipo de protector, con el fin de suministrar a los empleadores mayor información frente a la eficacia real de los protectores auditivos, para de esta manera, facilitarles el proceso de toma de decisiones en la compra.

El obtener resultados diferentes aplicando metodologías similares a las reportadas por los fabricantes, hace evidente la necesidad de contar con esquemas de certificación a modo de aval sobre el nivel de atenuación real de ruido en condiciones controladas.

En Colombia, se hace necesario profundizar sobre el conocimiento de los criterios de atenuación del ruido que utilizan los empleadores en el proceso de compra de los protectores auditivos, en relación con las necesidades particulares de las condiciones de trabajo, pero a su vez, se recomienda a las empresas, aplicar la metodología utilizada en el presente estudio, con el fin de lograr la mejor protección auditiva al menor costo posible.

\section{BIBLIOGRAFÍA}

1. Organización Internacional del Trabajo - ILO. www.ilo.org. [Online].; 2001 [cited 2013 Mayo 3. Available from: HYPERLINK http://www.ilo.org/wcmsp5/groups/public/---ed_protect/---protrav/---safework/ documents/normativeinstrument/wcms_112582.pdf.

2. Ministerio de la Protección Social - Colciencias. República de Colombia. Guía Metodológica para la elaboración de guías de atención integral en el Sistema General de Seguridad Social en Salud Colombiano. Primera ed. Torres JB, editor. Bogotá: Buenos \& Creativos S.A.S.; 2010.

3. Osteba - Servicio de Evaluación de Tecnologías Sanitaria. Departamento de Sanidad del Gobierno Vasco. [Online].; 1999 [cited 2013 Mayo 3. Available from: HYPERLINK http://www9.euskadi.net/sanidad/ osteba/datos/d_99-01_evaluacion_economica.pdf.

4. Colombia - Ministerio de la Protección Social. Guía de atención integral basada en la evidencia para hipoacusia neurosensorial inducida. Primera edición ed. Bogotá; 2006.

5. Colombia. Constitución Política de Colombia 1991. 13th ed. Oficial D, editor. Bogotá: Temis; 2011.

6. Colombia. Código sustantivo del trabajo y código procesal del trabajo y de la seguridad social. 26 th ed. Bogotá: Legis; 2010.

7. Drummond MF. Methods for the economic evaluation of health care programmes. Tercera Edición ed.: Oxford University Press; 2005.

8. Londoño J., Restrepo H., Corrales A., Mendoza F., Ortiz J. Hipoacusia neurosensorial por ruido industrial y solventes organicos en a Gerencia Complejo Barrancabermeja, 1977-1997. Rev.Nac.Salud Pública, p 94-120. 
9. Mancera Fernández M., Mancera Ruiz MT., Mancera Ruiz R., Mancera Ruiz JR. Seguridad e Higiene Industrial. Gestión de Riesgos. Primera Edición ed. Casallas OR, editor. Bogotá: Alfaomega; 2012.

10. OSHA EUROPEAN AGENCY FOR SAFETY AND HEALTH AT WORK. OSHA.EUROPA.EU. [Online].; 2012 [cited 2012 Mayo 25. Available from: HYPERLINK http://osha.europa.eu/es/topics/noise/what_is_noise_ html .

11. Instituto Colombiano de Normas Técnicas y Certificación. Norma Técnica Colombia NTC 2915 Bogotá: ICONTEC; 1991.

12. Sánchez Valenzuela, Mauricio. Instituto de Salud Pública Guía de Selección y Control de Equipos de Protección Auditiva. [En línea]. 2007. Fecha de acceso 25 de Febrero de 2013. Disponible en http://www. ispch.cl/salud_ocup/epp/epp/guia\%20de\%20seleccion\%20epa.\%20isp.\%20final.pdf.

13. Pascual, Francisco Javier. Recomendaciones para la selección y utilización de protectores auditivos [En línea]. Fecha de acceso 19 de Mayo de 2014. Disponible en http://www.segurancaetrabalho.com.br/ download/prot-audit-selecion.pdfx.

14. Ministerio de Trabajo y Asuntos Sociales de España. Instituto Nacional de Seguridad e Higiene en el Trabajo. NTP 284: Audiometría tonal liminar: exploraciones previas y vía aérea. [En línea]. Fecha de acceso 19 de Mayo de 2014. Disponible en http://www.insht.es/InshtWeb/Contenidos/Documentacion/ FichasTecnicas/NTP/Ficheros/201a300/ntp_284.pdf.

15. Solé, María Dolors. Programa de vigilancia de la salud de los trabajadores expuestos a ruido. 2005. [En línea] Fecha de acceso 19 de Mayo de 2014. Disponible en http://www.insht.es/InshtWeb/Contenidos/ Documentacion/TextosOnline/Rev_INSHT/2005/36/seccionUnicaTextCompl2.pdf. 